\title{
On Eventually Positive Solutions of Quasilinear Second-Order Neutral Differential Equations
}

\author{
Simona Fišnarová and Robert Mařík \\ Department of Mathematics, Mendel University in Brno, Zemědělská 1, 61300 Brno, Czech Republic \\ Correspondence should be addressed to Robert Mařík; marik@mendelu.cz
}

Received 17 February 2014; Accepted 16 April 2014; Published 13 May 2014

Academic Editor: Samir Saker

Copyright (C) 2014 S. Fišnarová and R. Mařík. This is an open access article distributed under the Creative Commons Attribution License, which permits unrestricted use, distribution, and reproduction in any medium, provided the original work is properly cited.

We study the second-order neutral delay differential equation $\left[r(t) \Phi_{\gamma}\left(z^{\prime}(t)\right)\right]^{\prime}+q(t) \Phi_{\beta}(x(\sigma(t)))=0$, where $\Phi_{\alpha}(t)=|t|^{\alpha-1} t, \alpha \geq 1$ and $z(t)=x(t)+p(t) x(\tau(t))$. Based on the conversion into a certain first-order delay differential equation we provide sufficient conditions for nonexistence of eventually positive solutions of two different types. We cover both cases of convergent and divergent integral $\int^{\infty} r^{-1 / \gamma}(t) \mathrm{d} t$. A suitable combination of our results yields new oscillation criteria for this equation. Examples are shown to exhibit that our results improve related results published recently by several authors. The results are new even in the linear case.

\section{Introduction}

In the paper we study the equation

$$
\begin{gathered}
{\left[r(t) \Phi_{\gamma}\left(z^{\prime}(t)\right)\right]^{\prime}+q(t) \Phi_{\beta}(x(\sigma(t)))=0,} \\
z(t)=x(t)+p(t) x(\tau(t)),
\end{gathered}
$$

where $\Phi_{\alpha}(t)=|t|^{\alpha-1} t, \alpha \geq 1$, is the power type nonlinearity. The coefficients $r$ and $p$ are subject to the usual conditions $r \in C^{1}\left(\left[t_{0}, \infty\right), \mathbb{R}^{+}\right), p \in C^{1}\left(\left[t_{0}, \infty\right), \mathbb{R}_{0}^{+}\right)$and the coefficient $q$ is positive, $q \in C\left(\left[t_{0}, \infty\right), \mathbb{R}^{+}\right)$.

We assume that $\lim _{t \rightarrow \infty} \tau(t)=\infty=\lim _{t \rightarrow \infty} \sigma(t)$,

$$
\sigma(\tau(t))=\tau(\sigma(t))
$$

and there exist numbers $p_{0} \geq 0$ and $\tau_{0}>0$ such that $p(t) \leq p_{0}$ and $\tau^{\prime}(t) \geq \tau_{0}$.

Under the solution of (1) we understand any differentiable function $x(t)$ which does not identically equal zero eventually, such that $r(t) \Phi_{\gamma}\left(z^{\prime}(t)\right)$ is differentiable and (1) holds for large $t$.

Following the widely accepted terminology, the solution of (1) is said to be oscillatory if it has infinitely many zeros tending to infinity. Equation (1) is said to be oscillatory if all its solutions are oscillatory. In the opposite case, that is, if there exists an eventually positive solution of (1), (1) is said to be nonoscillatory.

In the paper we study nonoscillatory solutions of (1). Since $x(t)$ is a solution of (1) if and only if $-x(t)$ is a solution of (1), we can focus our attention on positive solutions.

The paper is organized as follows. In the remaining part of the current section we summarize selected important facts related to (1) and trends in the oscillation theory of this equation. In Section 2 we summarize tools like inequalities and oscillation criteria used in the proofs of main results. The main results are presented in the next three sections. Results on eventually positive solutions are separated into Sections 3 and 4 according to different asymptotic behavior: $z^{\prime}(t)>0$ in Section 3 and $z^{\prime}(t)<0$ in Section 4. In both cases we provide an efficient condition which ensures that solutions of this type do not exist. Note that under some additional conditions (namely, divergence of integral (3) below) the results from Section 3 immediately yield also oscillation criteria. If (3) fails, we can formulate oscillation criteria using a suitable combination of results from Sections 3 and 4, as shown in Section 5. The results of the paper improve several recently published results even in the linear case. We discuss these improvements in detail in remarks and examples accompanying the main theorems. 
Neutral differential equation (1) as well as other related equations have been studied frequently in the literature. There are two main methods in the oscillation theory of (1). One of them is based on a modification of the classical Riccati substitution which is known to be a powerful tool in theory of second-order linear differential equations. Following this method, neutral equation (1) is in some sense considered as a perturbation of some second-order ordinary differential equation. An alternative approach, used for example, in a series of papers by Baculíková et al. [1-4] and Li [5] is based on the fact that it is possible to derive neutral first-order differential inequality for quasiderivative from (1) and the resulting inequality can be studied in the scope of theory elaborated for first-order delay differential inequalities. In this paper we use the later approach. The resulting theorems are sometimes referred to as comparison theorems for neutral differential equations.

Two main approaches are used to put the shift $\tau(t)$ in the differential term under the control. If $p(t)<1$, then (1) can be "majorized" (in the sense of the classical Sturm comparison theory, which however has no extension to delay equations) by a delay equation of the form (1) with $p(t)=0$. Oscillation criteria for second-order delay differential equations can be then used to conclude results for neutral equation (1) (see, e.g., [6-8]). An alternative approach deals with a suitable combination of (1) and the same equation with independent variable shifted from $t$ to $\tau(t)$. This approach, which is used also in our paper, does not require $p(t)<1$ but yields other restrictions, such as commutativity of the composition of delays (2).

Neglecting which method is used to study the oscillation of (1), it turns out that it is necessary to distinguish two cases: either

$$
\int^{\infty} \frac{1}{r^{1 / \gamma}(t)} \mathrm{d} t=\infty
$$

or

$$
\int^{\infty} \frac{1}{r^{1 / \gamma}(t)} \mathrm{d} t<\infty
$$

The absolute majority of oscillation results in the literature concerns case (3), since in this case the positive solutions of (1) exhibit simpler behavior than in case (4); see Lemma 5 below. Case (4) has been studied, for example, in [9-16]. Note that for this case it is typical that the oscillation criterion consists of two relatively independent conditions. One of them is used to eliminate positive solutions with $z^{\prime}(t)<0$; the other one to eliminate positive solutions with $z^{\prime}(t)>0$. There are also results which treat both cases $z^{\prime}(t)>0$ and $z^{\prime}(t)<0$ in one unified approach. However, following this approach a typical conclusion is weaker: the equation is proved to be almost oscillatory (all nonoscillatory solutions, if exist any, tend to zero). Note also that the paper [16] does not satisfy these rules (makes use of unified approach to both cases but concludes oscillation), but there are several inaccuracies in this paper; see $[10,17]$ for corrected version of $[16]$.
In this paper we essentially use the method from $[1,2]$ with a modification for case (4) presented in [12]. However, to keep the influence of each condition as transparent as possible we used different organization of the paper, as we explained above. The main improvement with respect to these papers is that we replace inequalities and estimates used in these papers by suitable parametrized versions depending on parameters $l$ and $\varphi$ (see below). This yields criteria with some degree of freedom and optimization with respect to the parameters which yields sharper results, as we carefully explain on examples of equations with proportional delay. A similar method where we use parameters $l$ and $\varphi$ to refine the widely used inequalities has been used in the recent paper [18].

Finally, note that [12] in fact deals with linear equations and the extension to nonlinear equations is suggested in Remark 11 at the end of the paper [12]. However here we use an advanced technique rather than the method suggested in [12].

\section{Preliminary Results}

In the paper we derive results related to the existence or nonexistence of certain equations and inequalities in terms of several parameters. The following two lemmas allow to find the values of the parameters, which yield sharpest results.

The function $h$ introduced in the following lemma plays a role in a formulation of oscillation criteria in the case $\beta \geq 1$.

Lemma 1. Let $\beta \geq 1$. The function

$$
h(x, y)=x^{\beta-1}+y\left(\frac{x}{x-1}\right)^{\beta-1}
$$

satisfies

$$
h(x, y) \geq h\left(1+y^{1 / \beta}, y\right)=\left(1+y^{1 / \beta}\right)^{\beta},
$$

for every $x>1$ and $y>0$.

Proof. It follows from the fact that

$$
\frac{\partial}{\partial x} h(x, y)=(\beta-1) x^{\beta-2}\left[1-\frac{y}{(x-1)^{\beta}}\right]
$$

and $h$ as the function of $x$ on $(1, \infty)$ attains its minimal value at the point $x=1+y^{1 / \beta}$ and

$$
\begin{aligned}
h\left(1+y^{1 / \beta}, y\right) & =\left(1+y^{1 / \beta}\right)^{\beta-1}+y^{1 / \beta}\left(1+y^{1 / \beta}\right)^{\beta-1} \\
& =\left(1+y^{1 / \beta}\right)^{\beta} .
\end{aligned}
$$

The following functions appear in the examples and allow to find the optimal values of the parameters which yield the sharpest result. 
Lemma 2. Let $c_{1}, c_{2}$ be positive numbers.

(i) The function $f(x)=x^{c_{1}} \ln \left(c_{2} / x\right)$ is increasing on ( 0 , $\left.c_{2} e^{-1 / c_{1}}\right)$, decreasing on $\left(c_{2} e^{-1 / c_{1}}, \infty\right)$, and satisfies

$$
f(x) \leq \frac{1}{c_{1} e} c_{2}^{c_{1}}
$$

on $(0, \infty)$ with the equality, if and only if $x=c_{2} e^{-1 / c_{1}}$.

(ii) The function $g(x)=x^{-c_{1}} \ln \left(x / c_{2}\right)$ is increasing on ( 0 , $\left.c_{2} e^{1 / c_{1}}\right)$, decreasing on $\left(c_{2} e^{1 / c_{1}}, \infty\right)$, and satisfies

$$
g(x) \leq \frac{1}{c_{1} e} c_{2}^{-c_{1}}
$$

on $(0, \infty)$ with the equality, if and only if $x=c_{2} e^{1 / c_{1}}$.

Proof. By a direct computation

$$
f^{\prime}(x)=x^{c_{1}-1}\left(c_{1} \ln \frac{c_{2}}{x}-1\right) .
$$

Hence $f(x)$ has a local maximum at the point $x_{0}=c_{2} e^{-1 / c_{1}}$ and the value of this local maximum is $c_{2}^{c_{1}} / c_{1} e$. Similarly,

$$
g^{\prime}(x)=x^{-1-c_{1}}\left(1-c_{1} \ln \frac{x}{c_{2}}\right) \text {, }
$$

and hence $g(x)$ has a local maximum at the point $x_{1}=c_{2} e^{1 / c_{1}}$ and the maximal value is $\left(1 / c_{1} e\right) c_{2}^{-c_{1}}$.

Lemma 3. Let $A \geq 0, B \geq 0, \beta \geq 1, l>1, l^{*}=l /(l-1)$. Then

$$
(A+B)^{\beta} \leq l^{\beta-1} A^{\beta}+\left(l^{*}\right)^{\beta-1} B^{\beta} .
$$

Proof. From the fact that the function $x^{\beta}$ is a convex function for $\beta \geq 1$ we have

$$
\left(\frac{1}{l} a+\frac{1}{l^{*}} b\right)^{\beta} \leq \frac{1}{l} a^{\beta}+\frac{1}{l^{*}} b^{\beta}
$$

for nonnegative $a$ and $b$. From here we obtain the desired inequality for $A=a / l$ and $B=b / l^{*}$.

Lemma 4. Let $\beta \geq 1$. The inequality

$$
l^{\beta-1} x^{\beta}(\sigma(t))+\left(l^{*}\right)^{\beta-1} p^{\beta}(\sigma(t)) x^{\beta}(\sigma(\tau(t))) \geq z^{\beta}(\sigma(t))
$$

holds for positive mutually conjugate numbers $l, l^{*}$ and every $t$ which satisfies $x(\sigma(t)) \geq 0$ and $x(\sigma(\tau(t))) \geq 0$.

Proof. It follows from Lemma 3, from the definition of $z(t)$ and from condition (2).

The following lemma is well known in theory of neutral differential equations. It states (among others) that if $x$ is an eventually positive solution, then $z^{\prime}$ is eventually of one sign and the negative sign of $z^{\prime}$ is excluded if (3) holds.
Lemma 5. Let $x(t)$ be an eventually positive solution of (1). The corresponding function $z(t)=x(t)+p(t) x(\tau(t))$ satisfies

$$
z(t)>0, \quad z^{\prime}(t)>0, \quad\left(r(t) \Phi_{\gamma}\left(z^{\prime}(t)\right)\right)^{\prime}<0
$$

eventually if (3) holds and either (16) or

$$
z(t)>0, \quad z^{\prime}(t)<0, \quad\left(r(t) \Phi_{\gamma}\left(z^{\prime}(t)\right)\right)^{\prime}<0
$$

eventually if (4) holds.

Proof. It follows from [7, Lemma 10] and from the proof of that lemma.

In the following lemma we summarize effective oscillation criteria for delay and advanced first-order equation which appear in the analysis of (1). Note that (iii) is sharper version of the related condition from [2, Lemma 4].

Lemma 6. Let $q(t) \geq 0$.

(i) If $\sigma(t)<t$ and

$$
\liminf _{t \rightarrow \infty} \int_{\sigma(t)}^{t} q(s) d s>\frac{1}{e},
$$

then

$$
y^{\prime}(t)+q(t) y(\sigma(t)) \leq 0
$$

has no eventually positive solution.

(ii) If $\sigma(t)>t$ and

$$
\liminf _{t \rightarrow \infty} \int_{t}^{\sigma(t)} q(s) d s>\frac{1}{e},
$$

then

$$
y^{\prime}(t)-q(t) y(\sigma(t)) \geq 0
$$

has no eventually positive solution.

(iii) Let $\sigma(t)<t, \alpha \in(0,1)$. If

$$
\int_{t_{0}}^{\infty} q(s) d s=\infty,
$$

then

$$
y^{\prime}(t)+q(t) y^{\alpha}(\sigma(t)) \leq 0
$$

has no eventually positive solution.

(iv) Let $\sigma(t)>t, \alpha \in(1, \infty)$. If

$$
\int_{t_{0}}^{\infty} q(s) d s=\infty,
$$

then

$$
y^{\prime}(t)-q(t) y^{\alpha}(\sigma(t)) \geq 0
$$

has no eventually positive solution.

Proof. See [9, Lemmas 2.1-2.4] and [19, Lemma 2.2.9]. Note that the original proof of condition (i) is due to [20] and the proofs of conditions (iii) and (iv) for equations are due to [21]. 


\section{Positive Solutions with $z^{\prime}(t)>0$ Eventually}

In this section we give sufficient conditions which exclude the possibility that the equation possesses an eventually positive solution $x(t)$ such that the corresponding function $z(t)$ is eventually increasing. Note that Lemma 5 excludes other types of eventually positive solutions if (3) holds. Hence if (3) holds as well, then the criteria from this section guarantee oscillation of (1).

Denote

$$
\begin{aligned}
Q(t ; \varphi) & =\min \{q(t), \varphi q(\tau(t))\}, \\
Q_{\eta}^{*}\left(t ; \varphi, t_{1}\right) & =Q(t ; \varphi)\left[\int_{t_{1}}^{\eta(t)} r^{-1 / \gamma}(s) \mathrm{d} s\right]^{\beta} .
\end{aligned}
$$

The following theorem allows us to relate positive solutions of (1) with a certain first-order neutral equation. This neutral equation can be further compared with a certain nonneutral differential equation. The form of this nonneutral differential equation depends on the fact whether the deviating argument $\tau(t)$ in the differential term is delay or advanced argument. If $\varphi=1, \eta \equiv \sigma$, and $l=2$, then Theorem 7 reduces to [2, Theorems 4, 5 and 6].

Theorem 7. Let $\beta \geq 1, \varphi>0, l>1$ and $\eta(t)$ a function which satisfies $\eta(t) \leq \sigma(t)$ and $\lim _{t \rightarrow \infty} \eta(t)=\infty$. Suppose that there exists a number $T>t_{0}$ and a solution $x(t)$ of (1) which satisfy

$$
x(t)>0, \quad z^{\prime}(t)>0 \quad \text { for } t \geq T .
$$

Let $t_{1}>T$ be such that

$$
\min \{\eta(\tau(t)), \eta(t)\}>T
$$

for every $t \geq t_{1}$ and let $t_{2} \geq t_{1}$ be such that $\eta(t) \geq t_{1}$ for $t \geq t_{2}$. Then the following statements are true.

(i) The inequality

$$
\begin{array}{r}
{\left[l^{\beta-1} w(t)+\frac{p_{0}^{\beta} \varphi}{\tau_{0}}\left(l^{*}\right)^{\beta-1} w(\tau(t))\right]^{\prime}} \\
+Q_{\eta}^{*}\left(t ; \varphi, t_{1}\right) w^{\beta / \gamma}(\eta(t)) \leq 0
\end{array}
$$

has a positive decreasing solution on $\left(t_{2}, \infty\right)$.

(ii) If $\tau(t) \geq t$, then

$$
y^{\prime}+Q_{\eta}^{*}\left(t ; \varphi, t_{1}\right) h^{-\beta / \gamma}\left(l, \frac{p_{0}^{\beta} \varphi}{\tau_{0}}\right) y^{\beta / \gamma}(\eta(t)) \leq 0
$$

has a positive solution on $\left(t_{2}, \infty\right)$.

(iii) If $\tau(t) \leq t$, then

$$
y^{\prime}+Q_{\eta}^{*}\left(t ; \varphi, t_{1}\right) h^{-\beta / \gamma}\left(l, \frac{p_{0}^{\beta} \varphi}{\tau_{0}}\right) y^{\beta / \gamma}\left(\tau^{-1}(\eta(t))\right) \leq 0
$$

has a positive solution on $\left(t_{2}, \infty\right)$.
Proof. Let $x(t)$ be a solution of (1) which satisfies $x(t)>0$ and $z^{\prime}(t)>0$ for $t \geq T$. Inequalities (15), $p(t) \leq p_{0}$, and $\eta(t) \leq \sigma(t)$ imply

$$
l^{\beta-1} x^{\beta}(\sigma(t))+\left(l^{*}\right)^{\beta-1} p_{0}^{\beta} x^{\beta}(\sigma(\tau(t))) \geq z^{\beta}(\eta(t))
$$

for $t \geq t_{1}$.

We shift (1) from $t$ to $\tau(t)$ and get

$$
\begin{aligned}
0= & \frac{1}{\tau^{\prime}(t)}\left[r(\tau(t)) \Phi_{\gamma}\left(z^{\prime}(\tau(t))\right)\right]^{\prime} \\
& +q(\tau(t)) \Phi_{\beta}(x(\sigma(\tau(t)))) \\
\geq & \frac{1}{\tau_{0}}\left[r(\tau(t)) \Phi_{\gamma}\left(z^{\prime}(\tau(t))\right)\right]^{\prime}+q(\tau(t)) \Phi_{\beta}(x(\sigma(\tau(t)))) .
\end{aligned}
$$

Substituting $\Phi_{\beta}(x(\sigma(\tau(t))))$ from this inequality and $\Phi_{\beta}(x(\sigma(t)))$ from (1) to (33) and using (26) we obtain

$$
\begin{aligned}
0 \geq & l^{\beta-1}\left[r(t) \Phi_{\gamma}\left(z^{\prime}(t)\right)\right]^{\prime}+\frac{\left(l^{*}\right)^{\beta-1} p_{0}^{\beta} \varphi}{\tau_{0}} \\
& \times\left[r(\tau(t)) \Phi_{\gamma}\left(z^{\prime}(\tau(t))\right)\right]^{\prime}+Q(t ; \varphi) z^{\beta}(\eta(t)),
\end{aligned}
$$

for $t \geq t_{1}$. Denoting $w(t)=r(t) \Phi_{\gamma}\left(z^{\prime}(t)\right)$ and using the obvious fact that $w$ is positive and decreasing on $\left(t_{1}, \infty\right)$ we have

$$
z(t)=\int_{t_{1}}^{t} w^{1 / \gamma}(s) r^{-1 / \gamma}(s) \mathrm{d} s \geq w^{1 / \gamma}(t) \int_{t_{1}}^{t} r^{-1 / \gamma}(s) \mathrm{d} s
$$

for $t \geq t_{1}$. Thus $w$ is an eventually positive and eventually decreasing solution of (30) and claim (i) is proved.

Denote

$$
y(t)=l^{\beta-1} w(t)+\frac{p_{0}^{\beta} \varphi}{\tau_{0}}\left(l^{*}\right)^{\beta-1} w(\tau(t)) .
$$

Since $w$ is a positive decreasing function, we have $w(t) \geq$ $w(\tau(t))$ for $t \leq \tau(t)$ and $w(t) \leq w(\tau(t))$ for $t \geq \tau(t)$. Hence if $t \leq \tau(t)$ we have

$$
y(t) \leq w(t)\left(l^{\beta-1}+\frac{p_{0}^{\beta} \varphi}{\tau_{0}}\left(l^{*}\right)^{\beta-1}\right)=w(t) h\left(l, \frac{p_{0}^{\beta} \varphi}{\tau_{0}}\right),
$$

where the function $h$ is defined by (5), and, if $t \geq \tau(t)$, then similarly

$$
y(t) \leq w(\tau(t)) h\left(l, \frac{p_{0}^{\beta} \varphi}{\tau_{0}}\right)
$$

Hence we have

$$
w^{\beta / \gamma}(\eta(t)) \geq h^{-\beta / \gamma}\left(l, \frac{p_{0}^{\beta} \varphi}{\tau_{0}}\right) y^{\beta / \gamma}(\eta(t)),
$$


if $t \leq \tau(t)$, and

$$
w^{\beta / \gamma}(\eta(t)) \geq h^{-\beta / \gamma}\left(l, \frac{p_{0}^{\beta} \varphi}{\tau_{0}}\right) y^{\beta / \gamma}\left(\tau^{-1}(\eta(t))\right)
$$

if $t \geq \tau(t)$. This and claim (i) prove claims (ii) and (iii) since in each case we have found an eventually positive solution $y$ of the corresponding inequality.

Remark 8. Note that in the proof of Theorem 7 we constructed the solutions of the inequalities (30), (31), and (32).

In the following corollary we give an efficient condition for nonexistence of the solutions mentioned in the points (ii) and (iii) of Theorem 7. According to Lemma 6 we distinguish the cases $\gamma=\beta$ and $\gamma \neq \beta$.

Corollary 9. Let $\gamma \geq \beta \geq 1$. Equation (1) has no solution $x(t)$ which satisfies

$$
x(t)>0, \quad z^{\prime}(t)>0 \quad \text { eventually }
$$

if there exists number $\varphi>0$ and a function $\eta(t)$ satisfying $\eta(t) \leq \sigma(t)$ and $\lim _{t \rightarrow \infty} \eta(t)=\infty$ such that one of the following conditions holds.

(i) $\eta(t)<t \leq \tau(t)$ and for every $T$ there exists $t_{1}>T$ such that

$$
\begin{aligned}
& \liminf _{t \rightarrow \infty} \int_{\eta(t)}^{t} Q_{\eta}^{*}\left(s ; \varphi, t_{1}\right) d s>\frac{1}{e}\left(1+\left(\frac{\varphi}{\tau_{0}}\right)^{1 / \beta} p_{0}\right)^{\beta^{2} / \gamma} \\
& \quad \text { if } \beta=\gamma \text {, and }
\end{aligned}
$$$$
\int_{t_{0}}^{\infty} Q_{\eta}^{*}\left(t ; \varphi, t_{1}\right) d t=\infty
$$

$$
\text { if } \beta<\gamma .
$$

(ii) $\eta(t)<\tau(t) \leq t$ and for every $T$ there exists $t_{1}>T$ such that

$$
\liminf _{t \rightarrow \infty} \int_{\tau^{-1}(\eta(t))}^{t} Q_{\eta}^{*}\left(s ; \varphi, t_{1}\right) d s>\frac{1}{e}\left(1+\left(\frac{\varphi}{\tau_{0}}\right)^{1 / \beta} p_{0}\right)^{\beta^{2} / \gamma}
$$

$$
\text { if } \beta=\gamma \text {, and (44) holds if } \beta<\gamma \text {. }
$$

Proof. It follows from Theorem 7 and Lemmas 6 and 1 .

In the following example we compare our results with the results of [1]. Note that in this example (3) holds and hence the results of this section ensure oscillation of the equation.

Example 10 (linear equation). Baculíková and Džurina studied in [1] differential equation

$$
\left(\sqrt{t}\left[x(t)+p_{0} x(\alpha t)\right]^{\prime}\right)^{\prime}+\frac{a}{t^{3 / 2}} x(\mu t)=0
$$

where $0<\mu<1, \alpha>0, a>0$, and obtained that the equation is oscillatory if either

$$
\alpha \geq 1, \quad 2 a \sqrt{\mu} \ln \frac{1}{\mu}>\frac{1}{e}\left(\alpha^{3 / 2}+p_{0} \sqrt{\alpha}\right)
$$

or

$$
0<\mu<\alpha \leq 1, \quad 2 a \sqrt{\mu} \ln \frac{\alpha}{\mu}>\frac{\alpha+p_{0}}{\alpha e} .
$$

We have $\gamma=\beta=1, \tau(t)=\alpha t, \sigma(t)=\mu t, r(t)=\sqrt{t}, q(t)=$ $a t^{-3 / 2}, q(\tau(t))=a(\alpha t)^{-3 / 2}$, and

$$
\int r^{-1 / \gamma}(t) \mathrm{d} t=\int t^{-1 / 2} \mathrm{~d} t=2 \sqrt{t}
$$

and hence (3) holds. Using Corollary 9 with $\eta(t)=\lambda t, \lambda \leq \mu$ and $\varphi=\alpha^{3 / 2}$ we have $Q(t)=q(t)$ and consequently,

$$
\begin{gathered}
Q_{\eta}^{*}\left(t ; \varphi, t_{1}\right)=\frac{a}{t^{3 / 2}}\left[2 \sqrt{\lambda} \sqrt{t}-2 \sqrt{t_{1}}\right], \\
\liminf _{t \rightarrow \infty} \int_{\eta(t)}^{t} Q_{\eta}^{*}\left(s ; \varphi, t_{1}\right) \mathrm{d} s=2 a \sqrt{\lambda} \ln \frac{1}{\lambda}, \\
\liminf _{t \rightarrow \infty} \int_{\tau^{-1}(\eta(t))}^{t} Q_{\eta}^{*}\left(s ; \varphi, t_{1}\right) \mathrm{d} s=2 a \sqrt{\lambda} \ln \frac{\alpha}{\lambda} .
\end{gathered}
$$

Hence, (46) is oscillatory if either

$$
\alpha \geq 1, \quad 2 a \sqrt{\lambda} \ln \frac{1}{\lambda}>\frac{1}{e}\left(1+\sqrt{\alpha} p_{0}\right)
$$

or

$$
0<\lambda<\alpha \leq 1, \quad 2 a \sqrt{\lambda} \ln \frac{\alpha}{\lambda}>\frac{1}{e}\left(1+\sqrt{\alpha} p_{0}\right) .
$$

Even in case $\mu=\lambda$, if we view the right hand sides as linear functions of $p_{0}$, we easily see from the slope and $y$-intercept of these lines that these conditions are sharper than those from [1]. Note that the fact that the equation is linear causes that the parameter $l$ does not have any influence on the sharpenes of these conditions, since the function $h$ from Lemma 1 is a constant function with respect to the first variable for $\beta=1$. Hence, the improvement with respect to the results from [1] is purely in the presence of the parameter $\varphi$ in the definition of the function $Q$. Figure 1 reveals also different asymptotic behavior of the right hand sides of (51) and (52) with respect to the corresponding constants from (47) and (48). Based on this fact we see that the improvement is significant especially if $\alpha$ is sufficiently far from 1 .

When looking for optimal conditions for oscillation of (46) it is easy to ensure that the case $\lambda=\mu$ is not optimal for every $\mu$. Really, if we replace inequality signs in (51) and (52) by equality signs and view the resulting equality as a formula which defines $a$ as a function of $\lambda$, we get $U$-shaped function with one local minimum (see Figure 2 ). Since $\lambda$ can be any positive number smaller than $\mu$, it turns out that the optimal choice for $\lambda$ is $\lambda=\mu$ on the decreasing part and $\lambda=$ $\lambda_{\text {max }}$ on the increasing part, where $\lambda_{\text {max }}$ is the point where 


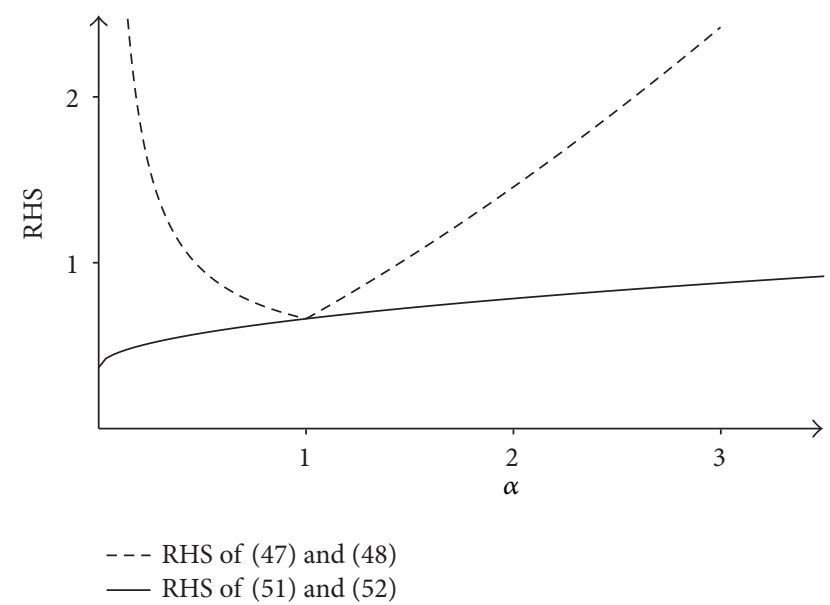

FIGURE 1: The graph of right hand sides (RHS) of (47) and (48) for $p_{0}=0.8$ compared to (51) and (52) as functions of $\alpha$.

the function $\sqrt{\lambda} \ln (\alpha / \lambda)$ attains its global maximum. Using Lemma 2, we have $\lambda_{\max }=\alpha / e^{2}$ and $\sqrt{\lambda} \ln (\alpha / \lambda) \leq(2 \sqrt{\alpha} / e)$. Hence we get that the equation is oscillatory if

$$
\begin{aligned}
& a>a_{\text {crit. }} \\
& := \begin{cases}\frac{1}{4}\left(1+\sqrt{\alpha} p_{0}\right) & \text { for } \alpha \geq 1, \mu \geq \frac{1}{e^{2}}, \\
\frac{1}{4 \sqrt{\alpha}}\left(1+\sqrt{\alpha} p_{0}\right) & \text { for } \alpha \leq 1, \mu \geq \frac{\alpha}{e^{2}}, \\
\frac{1}{2 e \sqrt{\mu} \ln (1 / \mu)}\left(1+\sqrt{\alpha} p_{0}\right) & \text { for } \alpha \geq 1, \mu \leq \frac{1}{e^{2}}, \\
\frac{1}{2 e \sqrt{\mu} \ln (\alpha / \mu)}\left(1+\sqrt{\alpha} p_{0}\right) & \text { for } \alpha \leq 1, \mu \leq \frac{\alpha}{e^{2}} .\end{cases}
\end{aligned}
$$

From the graphical point of view these conditions arise from (51) and (52) by isolating $a$ and replacing the increasing part of the resulting curve by a constant function; see Figure 2 for more details and for comparison with the oscillation constant resulting from (48).

Example 11 (half-linear equation). Consider the differential equation

$$
\begin{array}{r}
\left(t^{2}\left[(x(t)+(3+\sin t) x(\alpha t))^{\prime}\right]^{3}\right)^{\prime}+\frac{b}{t^{2}} x^{3}(\mu t)=0, \\
\mu<1, \quad a>0 .
\end{array}
$$

We have $\beta=\gamma=3, \tau(t)=\alpha t, \sigma(t)=\mu t, p_{0}=4, \tau_{0}=\alpha$, $r(t)=t^{2}, q(t)=b / t^{2}, q(\tau(t))=b /(\alpha t)^{2}$ and,

$$
\int r^{-1 / \gamma}(t) \mathrm{d} t=\int t^{-2 / 3} \mathrm{~d} t=3 t^{1 / 3}
$$

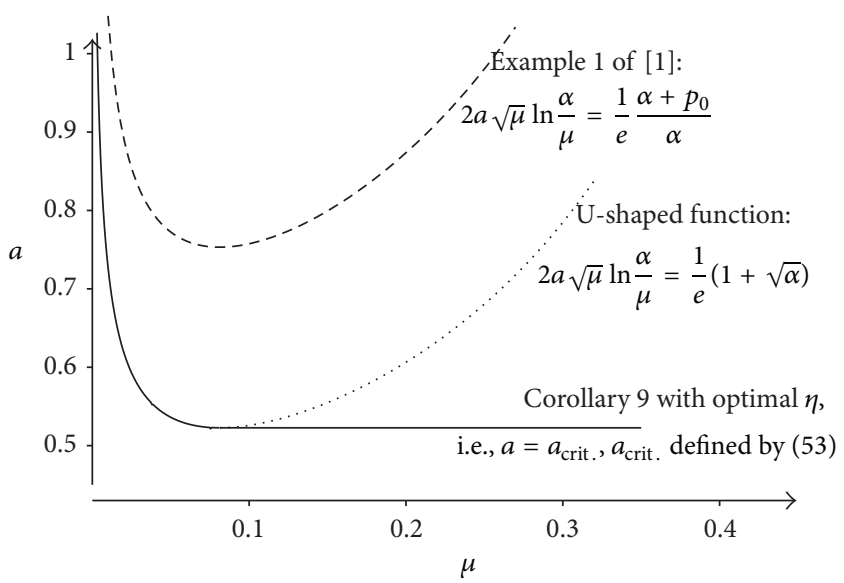

Figure 2: A comparison of the lower bounds for the coefficient $a$ which guarantee oscillation of (46) for different values of $\mu$. Parameters used for the graphs are $\alpha=0.6$ and $p_{0}=0.8$.

and hence condition (3) holds. Using results of Baculíková and Džurina [2, Corollaries 3 and 4] we obtain that (54) is oscillatory if

$$
\alpha \geq 1, \quad 27 e b \mu \ln \frac{1}{\mu}>4 \alpha^{2}\left(1+\frac{4^{3}}{\alpha}\right)
$$

or

$$
0<\mu<\alpha \leq 1, \quad 27 e b \mu \ln \frac{\alpha}{\mu}>4\left(1+\frac{4^{3}}{\alpha}\right) .
$$

Using Corollary 9 with $\eta(t)=\lambda t, \lambda \leq \mu$ and $\varphi=\alpha^{2}$ we have $Q(t ; \varphi)=q(t)$ and consequently,

$$
Q_{\eta}^{*}\left(t ; \varphi, t_{1}\right)=\frac{27 b}{t^{2}}\left[\lambda^{1 / 3} t^{1 / 3}-t_{1}^{1 / 3}\right]^{3} .
$$

Hence,

$$
\begin{gathered}
\liminf _{t \rightarrow \infty} \int_{\eta(t)}^{t} Q_{\eta}^{*}\left(s ; \varphi, t_{1}\right) \mathrm{d} s=27 b \lambda \ln \frac{1}{\lambda}, \\
\liminf _{t \rightarrow \infty} \int_{\tau^{-1}(\eta(t))}^{t} Q_{\eta}^{*}\left(s ; \varphi, t_{1}\right) \mathrm{d} s=27 b \lambda \ln \frac{\alpha}{\lambda}
\end{gathered}
$$

and (54) is oscillatory if

$$
\alpha \geq 1, \quad 27 e b \lambda \ln \frac{1}{\lambda}>\left(1+4 \alpha^{1 / 3}\right)^{3}
$$

or

$$
0<\lambda<\alpha \leq 1, \quad 27 e b \lambda \ln \frac{\alpha}{\lambda}>\left(1+4 \alpha^{1 / 3}\right)^{3} .
$$

If $\lambda=\mu$, the comparison of our lower bound $\left(1+4 \alpha^{1 / 3}\right)^{3}$ and the upper bound $4\left(1+4^{3} / \alpha\right)$ if $\alpha<1$ and $4 \alpha^{2}\left(1+4^{3} / \alpha\right)$ if $\alpha>1$ is on Figure 3. Note that in contrast to the linear case, both curves do not intersect at $\alpha=1$. 


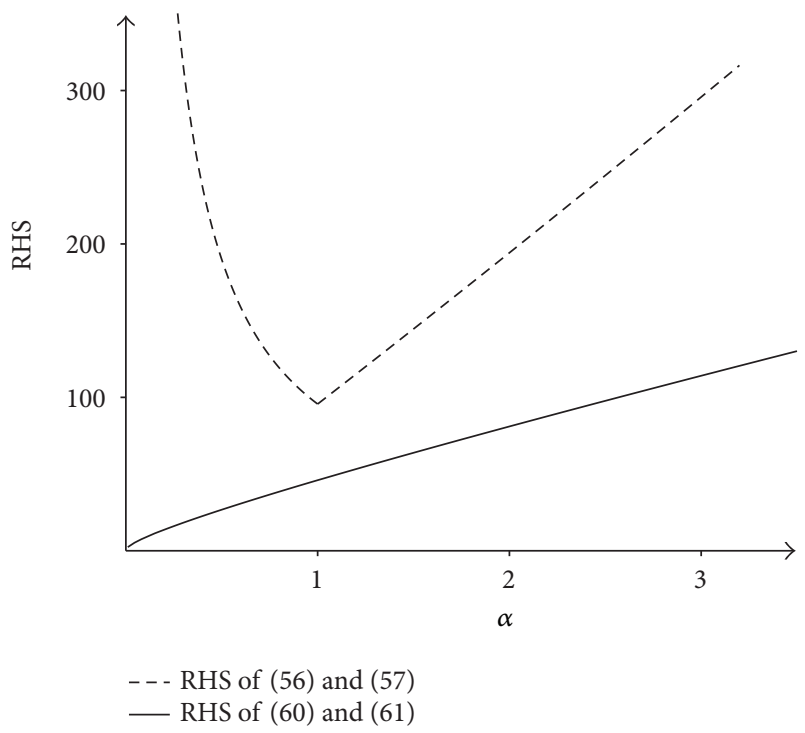

Figure 3: The graph of right hand sides (RHS) of (56) and (57) for $p_{0}=4$ compared to (60) and (61) as functions of $\alpha$.

The function $f(\lambda)=\lambda \ln (\alpha / \lambda)$ is positive on the interval $(0, \alpha)$ and it follows from Lemma 1 that the maximal value of $f(\lambda)$ is $\alpha / e$ at the point $\lambda=\alpha / e$. Hence, (54) is oscillatory if

$$
\begin{aligned}
& b>b_{\text {crit. }} \\
& := \begin{cases}\frac{1}{27}\left(1+4 \alpha^{1 / 3}\right)^{3} & \text { for } \alpha \geq 1, \mu \geq \frac{1}{e}, \\
\frac{1}{27 \alpha}\left(1+4 \alpha^{1 / 3}\right)^{3} & \text { for } \alpha \leq 1, \mu \geq \frac{\alpha}{e}, \\
\frac{1}{27 e \mu \ln (1 / \mu)}\left(1+4 \alpha^{1 / 3}\right)^{3} & \text { for } \alpha \geq 1, \mu \leq \frac{1}{e}, \\
\frac{1}{27 e \mu \ln (\alpha / \mu)}\left(1+4 \alpha^{1 / 3}\right)^{3} & \text { for } \alpha \leq 1, \mu \leq \frac{\alpha}{e} .\end{cases}
\end{aligned}
$$

Figure 4 shows how the critical constant which ensures oscillation of (54) is improved with respect to the results of [2] (dashed line) for various values of the delay $\mu$. For readers convenience we graphed also a dotted curve which is only partial improvement of [2]: the values of $l$ and $\eta$ are chosen as in [2] and the value of $\varphi$ (which plays role in $Q$ ) is choosen to equal to $q(t) / q(\tau(t))$.

The following theorem and corollary are variants of Theorem 7 and Corollary 9 for sublinear case $\beta \leq 1$.

Theorem 12. Let $\beta \leq 1, \varphi>0$, and $\eta(t)$ a function which satisfies $\eta(t) \leq \sigma(t)$ and $\lim _{t \rightarrow \infty} \eta(t)=\infty$. Suppose that there exists a number $T>t_{0}$ and a solution $x(t)$ of (1) which satisfy

$$
x(t)>0, \quad z^{\prime}(t)>0 \quad \text { for } t \geq T .
$$

Let $t_{1}>T$ be such that

$$
\min \{\eta(\tau(t)), \eta(t)\}>T
$$

for every $t \geq t_{1}$ and let $t_{2} \geq t_{1}$ be such that $\eta(t) \geq t_{1}$ for $t \geq t_{2}$. Then the following statements are true.

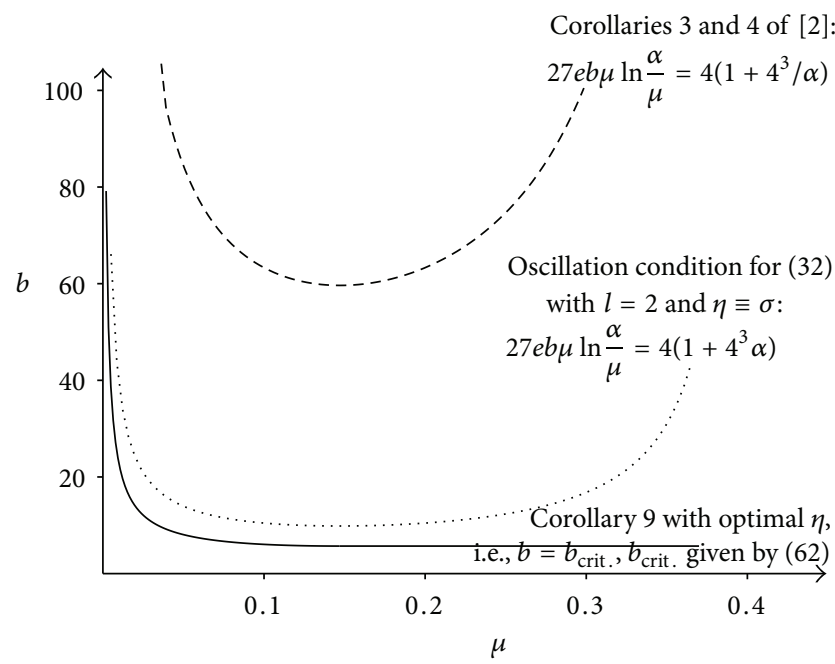

FIGURE 4: A comparison of the lower bounds for the coefficient $b$ which guarantee oscillation of (54) for different values of $\mu$. Parameters are $\alpha=0.4$ and $p_{0}=4$.

(i) The inequality

$$
\left[w(t)+\frac{p_{0}^{\beta} \varphi}{\tau_{0}} w(\tau(t))\right]^{\prime}+Q_{\eta}^{*}\left(t ; \varphi, t_{1}\right) w^{\beta / \gamma}(\eta(t)) \leq 0
$$

has a positive decreasing solution on $\left(t_{2}, \infty\right)$.

(ii) If $\tau(t) \geq t$, then

$$
y^{\prime}+Q_{\eta}^{*}\left(t ; \varphi, t_{1}\right)\left(\frac{\tau_{0}}{\tau_{0}+p_{0}^{\beta} \varphi}\right)^{\beta / \gamma} y^{\beta / \gamma}(\eta(t)) \leq 0
$$

has a positive solution on $\left(t_{2}, \infty\right)$.

(iii) If $\tau(t) \leq t$, then

$$
y^{\prime}+Q_{\eta}^{*}\left(t ; \varphi, t_{1}\right)\left(\frac{\tau_{0}}{\tau_{0}+p_{0}^{\beta} \varphi}\right)^{\beta / \gamma} y^{\beta / \gamma}\left(\tau^{-1}(\eta(t))\right) \leq 0
$$

has a positive solution on $\left(t_{2}, \infty\right)$.

Proof. The proof is the same as the proof of Theorem 7 where we formally put $l=l^{*}=1$ and use

$$
(A+B)^{\beta} \leq A^{\beta}+B^{\beta}
$$

instead of Lemma 3.

Corollary 13. Let $\beta \leq 1, \gamma \geq \beta$. Equation (1) has no solution $x(t)$ which satisfies

$$
x(t)>0, \quad z^{\prime}(t)>0 \quad \text { eventually }
$$

if there exists $\varphi>0$ and a function $\eta(t)$ satisfying $\eta(t) \leq \sigma(t)$ and $\lim _{t \rightarrow \infty} \eta(t)=\infty$ such that one of the following conditions holds. 
(i) $\eta(t)<t \leq \tau(t)$ and for every $T$ there exists $t_{1}>T$ such that

$$
\liminf _{t \rightarrow \infty} \int_{\eta(t)}^{t} Q_{\eta}^{*}\left(s ; \varphi, t_{1}\right) d s>\frac{1}{e}\left(\frac{\tau_{0}+p_{0}^{\beta} \varphi}{\tau_{0}}\right)^{\beta / \gamma}
$$

$$
\text { if } \beta=\gamma \text {, and (44) holds if } \beta<\gamma \text {. }
$$

(ii) $\eta(t)<\tau(t) \leq t$ and for every $T$ there exists $t_{1}>T$ such that

$$
\begin{gathered}
\liminf _{t \rightarrow \infty} \int_{\tau^{-1}(\eta(t))}^{t} Q_{\eta}^{*}\left(s ; \varphi, t_{1}\right) d s>\frac{1}{e}\left(\frac{\tau_{0}+p_{0}^{\beta} \varphi}{\tau_{0}}\right)^{\beta / \gamma} \\
\text { if } \beta=\gamma \text {, and (44) holds if } \beta<\gamma .
\end{gathered}
$$

Proof. It follows from Theorem 12 and Lemmas 6 and 1.

Remark 14. If $\varphi=1$ and $\eta(t)=\sigma(t)$, then Theorem 12 reduces to [2, Theorems 1, 2, and 3] and Corollary 13 reduces to [2, Corollaries 1 and 2].

\section{Positive Solutions with $z^{\prime}(t)<0$ Eventually}

In this section we modify the methods from previous section for positive solutions $x(t)$ which satisfy $z^{\prime}(t)<0$ eventually. Troughout this section we will suppose that (4) holds, since if it fails, then evetually positive solutions with $z^{\prime}(t)<0$ eventually do not exist.

The function $Q_{\zeta}^{\star}$ defined by the relation

$$
Q_{\zeta}^{\star}(t ; \varphi)=Q(t ; \varphi)\left[\int_{\zeta(t)}^{\infty} r^{-1 / \gamma}(s) \mathrm{d} s\right]^{\beta}
$$

and the following Theorem 15 are the corresponding modifications of the function $Q_{\eta}^{*}$ and Theorem 7 .

Theorem 15. Let $\beta \geq 1, \varphi>0, l>1$, and let $\zeta(t)$ be a function which satisfies $\zeta(t) \geq \sigma(t)$. Suppose that there exists a number $T>t_{0}$ and a solution $x(t)$ of (1) which satisfy

$$
x(t)>0, \quad z^{\prime}(t)<0 \quad \text { for } t \geq T .
$$

Let $t_{1}>T$ be such that

$$
\min \{\sigma(t), \sigma(\tau(t))\}>T
$$

for every $t \geq t_{1}$. Then the following statements are true.

(i) The inequality

$$
\begin{gathered}
{\left[l^{\beta-1} u(t)+\frac{p_{0}^{\beta} \varphi}{\tau_{0}}\left(l^{*}\right)^{\beta-1} u(\tau(t))\right]^{\prime}} \\
-Q_{\zeta}^{\star}(t ; \varphi) u^{\beta / \gamma}(\zeta(t)) \geq 0
\end{gathered}
$$

has a positive increasing solution on $\left(t_{1}, \infty\right)$.

(ii) If $\tau(t) \leq t$, then

$$
y^{\prime}-Q_{\zeta}^{\star}(t ; \varphi) h^{-\beta / \gamma}\left(l, \frac{p_{0}^{\beta} \varphi}{\tau_{0}}\right) y^{\beta / \gamma}(\zeta(t)) \geq 0
$$

(iii) If $\tau(t) \geq t$, then

$$
y^{\prime}-Q_{\zeta}^{\star}(t ; \varphi) h^{-\beta / \gamma}\left(l, \frac{p_{0}^{\beta} \varphi}{\tau_{0}}\right) y^{\beta / \gamma}\left(\tau^{-1}(\zeta(t))\right) \geq 0
$$

has a positive solution on $\left(t_{1}, \infty\right)$.

Proof. Let $x$ be a solution of (1) which satisfies $x(t)>0$ and $z^{\prime}(t)<0$ for $t>T$. Inequalities (15), $p(t) \leq p_{0}$, and $\zeta(t) \geq \sigma(t)$ imply

$$
l^{\beta-1} x^{\beta}(\sigma(t))+\left(l^{*}\right)^{\beta-1} p_{0}^{\beta} x^{\beta}(\sigma(\tau(t))) \geq z^{\beta}(\zeta(t)) .
$$

Combining this inequality with (1) and (1) shifted from $t$ to $\tau(t)$, similarly as in the proof of Theorem 7 , we obtain

$$
\begin{aligned}
0 \geq & l^{\beta-1}\left[r(t) \Phi_{\gamma}\left(z^{\prime}(t)\right)\right]^{\prime}+\frac{\left(l^{*}\right)^{\beta-1} p_{0}^{\beta} \varphi}{\tau_{0}} \\
& \times\left[r(\tau(t)) \Phi_{\gamma}\left(z^{\prime}(\tau(t))\right)\right]^{\prime}+Q(t ; \varphi) z^{\beta}(\zeta(t))
\end{aligned}
$$

for $t \geq t_{1}$. The function $w$ defined by $w(t)=r(t) \Phi_{\gamma}\left(z^{\prime}(t)\right)$ is negative and decreasing. Hence for $s \geq t$ we have

$$
\begin{gathered}
r(s) \Phi_{\gamma}\left(z^{\prime}(s)\right) \leq r(t) \Phi_{\gamma}\left(z^{\prime}(t)\right) \\
z^{\prime}(s) \leq z^{\prime}(t) \Phi_{\gamma}^{-1}\left(\frac{r(t)}{r(s)}\right)
\end{gathered}
$$

and hence

$$
z(l)-z(t) \leq \Phi_{\gamma}^{-1}(r(t)) z^{\prime}(t) \int_{t}^{l} r^{-1 / \gamma}(s) \mathrm{d} s .
$$

Since $\lim _{l \rightarrow \infty} z(l) \geq 0$ we have

$$
-z(t) \leq \Phi_{\gamma}^{-1}(r(t)) z^{\prime}(t) \int_{t}^{\infty} r^{-1 / \gamma}(s) \mathrm{d} s
$$

which implies

$$
z^{\beta}(\zeta(t)) \geq(-w(\zeta(t)))^{\beta / \gamma}\left[\int_{\zeta(t)}^{\infty} r^{-1 / \gamma}(s) \mathrm{d} s\right]^{\beta} .
$$

Combining this inequality with (79) and multiplying by -1 we find that $u(t)=-w(t)$ is a positive and increasing solution of (75). Claim (i) is proved. Denote

$$
y(t)=l^{\beta-1} w(t)+\frac{p_{0}^{\beta} \varphi}{\tau_{0}}\left(l^{*}\right)^{\beta-1} w(\tau(t)) .
$$

Since $u(t)$ is positive and increasing, we have $u(\tau(t)) \leq u(t)$ for $\tau(t) \leq t$ and $u(\tau(t)) \geq u(t)$ for $\tau(t) \geq t$. Hence, if $\tau(t) \leq t$, we have

$$
y(t) \leq u(t) h\left(l, \frac{p_{0}^{\beta} \varphi}{\tau_{0}}\right)
$$

which implies

$$
u^{\beta / \gamma}(\zeta(t)) \geq h^{-\beta / \gamma}\left(l, \frac{p_{0}^{\beta} \varphi}{\tau_{0}}\right) y^{\beta / \gamma}(\zeta(t)) .
$$


Analogously, if $\tau(t) \geq t$,

$$
y(t) \leq u(\tau(t)) h\left(l, \frac{p_{0}^{\beta} \varphi}{\tau_{0}}\right)
$$

which implies

$$
u^{\beta / \gamma}(\zeta(t)) \geq h^{-\beta / \gamma}\left(l, \frac{p_{0}^{\beta} \varphi}{\tau_{0}}\right) y^{\beta / \gamma}\left(\tau^{-1}(\zeta(t))\right) .
$$

Claims (ii) and (iii) then follow from (i) and positivity of $y$.

Corollary 16. Let $\beta \geq 1$ and $\beta \geq \gamma$. Equation (1) has no solution $x(t)$ which satisfies

$$
x(t)>0, \quad z^{\prime}(t)<0 \quad \text { eventually }
$$

if there exists $\varphi>0$ and a function $\zeta(t)$ satisfying $\zeta(t) \geq \sigma(t)$ such that one of the following conditions holds.

(i) $\tau(t) \leq t<\zeta(t)$ and

$$
\begin{aligned}
& \liminf _{t \rightarrow \infty} \int_{t}^{\zeta(t)} Q_{\zeta}^{\star}(s ; \varphi) d s>\frac{1}{e}\left(1+\left(\frac{\varphi}{\tau_{0}}\right)^{1 / \beta} p_{0}\right)^{\beta^{2} / \gamma} \\
& \quad \text { if } \beta=\gamma \text {, and }
\end{aligned}
$$

$$
\int_{t_{0}}^{\infty} Q_{\zeta}^{\star}(t ; \varphi) d t=\infty
$$

if $\beta>\gamma$.

(ii) $t \leq \tau(t)<\zeta(t)$ and

$$
\liminf _{t \rightarrow \infty} \int_{t}^{\tau^{-1}(\zeta(t))} Q_{\zeta}^{\star}(s ; \varphi) d s>\frac{1}{e}\left(1+\left(\frac{\varphi}{\tau_{0}}\right)^{1 / \beta} p_{0}\right)^{\beta^{2} / \gamma}
$$

$$
\text { if } \beta=\gamma \text {, and (91) holds if } \beta>\gamma \text {. }
$$

Proof. It follows from Theorem 15 and Lemmas 6 and 1.

In a similar way as in Theorem 12 and Corollary 13, if we suppose $\beta \leq 1$ in Theorem 15 and Corollary 16 and use inequality (68) instead of inequality from Lemma 3, we get the following statements.

Theorem 17. Let $\beta \leq 1, \varphi>0$, and $\zeta(t)$ a function which satisfies $\zeta(t) \geq \sigma(t)$. Suppose that there exists a number $T>t_{0}$ and a solution $x(t)$ of (1) which satisfy

$$
x(t)>0, \quad z^{\prime}(t)<0 \quad \text { for } t \geq T .
$$

Let $t_{1}>T$ be such that

$$
\min \{\sigma(t), \sigma(\tau(t))\}>T
$$

for every $t \geq t_{1}$. Then the following statements are true. (i) The inequality

$$
\left[u(t)+\frac{p_{0}^{\beta} \varphi}{\tau_{0}} u(\tau(t))\right]^{\prime}-Q_{\zeta}^{\star}(t ; \varphi) u^{\beta / \gamma}(\zeta(t)) \geq 0
$$

has a positive increasing solution on $\left(t_{1}, \infty\right)$.

(ii) If $\tau(t) \leq t$, then

$$
y^{\prime}-Q_{\zeta}^{\star}(t ; \varphi)\left(\frac{\tau_{0}}{\tau_{0}+p_{0}^{\beta} \varphi}\right)^{\beta / \gamma} y^{\beta / \gamma}(\zeta(t)) \geq 0
$$

has a positive solution on $\left(t_{1}, \infty\right)$.

(iii) If $\tau(t) \geq t$, then

$$
y^{\prime}-Q_{\zeta}^{\star}(t ; \varphi)\left(\frac{\tau_{0}}{\tau_{0}+p_{0}^{\beta} \varphi}\right)^{\beta / \gamma} y^{\beta / \gamma}\left(\tau^{-1}(\zeta(t))\right) \geq 0
$$

has a positive solution on $\left(t_{1}, \infty\right)$.

Corollary 18. Let $\beta \leq 1, \gamma \leq \beta$. Equation (1) has no solution $x(t)$ which satisfies

$$
x(t)>0 \quad z^{\prime}(t)<0 \quad \text { eventually }
$$

if there exists $\varphi>0$ and a function $\zeta(t)$ satisfying $\eta(t) \geq \sigma(t)$ such that one of the following conditions holds.

(i) $\tau(t) \leq t<\zeta(t)$ and

$$
\liminf _{t \rightarrow \infty} \int_{t}^{\zeta(t)} Q_{\zeta}^{\star}(s ; \varphi) d s>\frac{1}{e}\left(\frac{\tau_{0}+p_{0}^{\beta} \varphi}{\tau_{0}}\right)^{\beta / \gamma}
$$

$$
\text { if } \beta=\gamma \text {, and (91) holds if } \beta>\gamma \text {. }
$$

(ii) $t \leq \tau(t)<\zeta(t)$ and

$$
\liminf _{t \rightarrow \infty} \int_{t}^{\tau^{-1}(\zeta(t))} Q_{\zeta}^{\star}(s ; \varphi) d s>\frac{1}{e}\left(\frac{\tau_{0}+p_{0}^{\beta} \varphi}{\tau_{0}}\right)^{\beta / \gamma}
$$

$$
\text { if } \beta=\gamma \text {, and (91) holds if } \beta>\gamma \text {. }
$$

\section{Oscillation Criteria If (4) Holds}

As we explained before, it follows from Lemma 5 that if (3) holds, then the criteria from Section 3 are in fact oscillation criteria. If (3) fails and (4) holds, then the set of all possible eventually positive solutions is more comprehensive and may contain also solution which satisfy $z^{\prime}(t)<0$ eventually. Hence to ensure oscillation of (1) in the case (4) we have to eliminate both cases; criteria from both Sections 3 and 4 apply. For example, in the half-linear case $\beta=\gamma$, (1) is oscillatory if either conditions

$$
\text { (4), (43), and (92) hold if } \tau(t) \geq t \text {, }
$$

or

$$
\text { (4), (45) and (90) hold if } \tau(t) \leq t \text {; }
$$

see the example below. 
Example 19. Consider the equation

$$
\left(t^{3 / 2}\left(x(t)+p_{0} x(\alpha t)\right)^{\prime}\right)^{\prime}+\frac{a}{\sqrt{t}} x(\mu t)=0, \quad a>0 .
$$

We have $\gamma=\beta=1, \tau(t)=\alpha t, \sigma(t)=\mu t, r(t)=t^{3 / 2}, q(t)=$ $a t^{-1 / 2}, q(\tau(t))=a(\alpha t)^{-1 / 2}$. We will apply Corollaries 9 and 16 with $\eta(t)=\lambda_{1} t, \zeta(t)=\lambda_{2} t, \lambda_{1} \leq \mu \leq \lambda_{2}$ and $\varphi=\alpha^{1 / 2}$. We have $Q(t)=q(t)$. Since

$$
\int r^{-1 / \gamma}(t) \mathrm{d} t=\int t^{-3 / 2} \mathrm{~d} t=-2 t^{-1 / 2}
$$

condition (4) holds. Next we have

$$
\begin{gathered}
Q_{\eta}^{*}\left(t ; \varphi, t_{1}\right)=-2 a\left(\sqrt{\lambda_{1}} t^{-1}-\sqrt{t_{1}} t^{-1 / 2}\right), \\
Q_{\zeta}^{\star}(t ; \varphi)=-2 a t^{-1 / 2}\left(\lim _{t \rightarrow \infty} t^{-1 / 2}-\left(\lambda_{2} t\right)^{-1 / 2}\right) \\
=2 a \lambda^{-1 / 2} t^{-1} .
\end{gathered}
$$

Consequently, if $\lambda_{1}<1 \leq \alpha<\lambda_{2}$, then (43) and (92) give

$$
\begin{aligned}
& \liminf _{t \rightarrow \infty} \int_{\eta(t)}^{t} Q_{\eta}^{*}\left(s ; \varphi, t_{1}\right) \mathrm{d} s \\
& =-2 a \sqrt{\lambda_{1}} \ln \frac{1}{\lambda_{1}}+2 a \sqrt{t_{1}}\left(1-\sqrt{\lambda_{1}}\right) \lim _{t \rightarrow \infty} \sqrt{t} \\
& =\infty, \\
& \liminf _{t \rightarrow \infty} \int_{t}^{\tau^{-1}(\zeta(t))} Q_{\zeta}^{\star}(s ; \varphi) \mathrm{d} s=\frac{2 a}{\sqrt{\lambda_{2}}} \ln \frac{\lambda_{2}}{\alpha} .
\end{aligned}
$$

If $\lambda_{1}<\alpha \leq 1<\lambda_{2}$, then (45) and (90) give

$$
\begin{aligned}
& \liminf _{t \rightarrow \infty} \int_{\tau^{-1}(\eta(t))}^{t} Q_{\eta}^{*}\left(s ; \varphi, t_{1}\right) \mathrm{d} s \\
& \quad=-2 a \sqrt{\lambda_{1}} \ln \frac{\alpha}{\lambda_{1}}+2 a \sqrt{t_{1}}\left(1-\sqrt{\frac{\lambda_{1}}{\alpha}}\right) \lim _{t \rightarrow \infty} \sqrt{t} \\
& \quad=\infty
\end{aligned}
$$$$
\liminf _{t \rightarrow \infty} \int_{t}^{\zeta(t)} Q_{\zeta}^{\star}(s ; \varphi) \mathrm{d} s=\frac{2 a}{\sqrt{\lambda}_{2}} \ln \lambda_{2} .
$$

Hence, (103) is oscillatory if

$$
\lambda_{1}<1 \leq \alpha<\lambda_{2}, \quad \frac{2 a}{\sqrt{\lambda}_{2}} \ln \frac{\lambda_{2}}{\alpha}>\frac{1}{e}\left(1+\frac{p_{0}}{\sqrt{\alpha}}\right)
$$

or

$$
\lambda_{1}<\alpha \leq 1<\lambda_{2}, \quad \frac{2 a}{\sqrt{\lambda}_{2}} \ln \lambda_{2}>\frac{1}{e}\left(1+\frac{p_{0}}{\sqrt{\alpha}}\right) .
$$

The function $g\left(\lambda_{2}\right)=\lambda_{2}^{-1 / 2} \ln \left(\lambda_{2} / \alpha\right)$ is positive on the interval $(\alpha, \infty)$ and it follows from Lemma 1 that the maximal value of $g\left(\lambda_{2}\right)$ is $2 /(e \sqrt{\alpha})$ at the point $\alpha e^{2}$. Hence, (103) is oscillatory if

$$
\begin{aligned}
& a>a_{\text {crit. }} \\
& := \begin{cases}\frac{\sqrt{\alpha}}{4}\left(1+\frac{p_{0}}{\sqrt{\alpha}}\right) & \text { for } \alpha \geq 1, \mu \leq \alpha e^{2}, \\
\frac{1}{4}\left(1+\frac{p_{0}}{\sqrt{\alpha}}\right) & \text { for } \alpha \leq 1, \mu \leq e^{2}, \\
\frac{\sqrt{\mu}}{2 e \ln \left(\frac{\mu}{\alpha}\right)}\left(1+\frac{p_{0}}{\sqrt{\alpha}}\right) & \text { for } \alpha \geq 1, \mu \geq \alpha e^{2}, \\
\frac{\sqrt{\mu}}{2 e \ln \mu}\left(1+\frac{p_{0}}{\sqrt{\alpha}}\right) & \text { for } \alpha \leq 1, \mu \geq e^{2} .\end{cases}
\end{aligned}
$$

\section{Conclusion}

In the paper we derived asymptotic results for neutral quasilinear equation (1). Note that this equation covers several types of second-order differential equations studied in the literature, namely, the linear and half-linear second-order differential equations.

Using the comparison method we derived sufficient conditions for nonexistence of eventually positive solutions with various asymptotic behaviors. Additional assumptions (such as (3)) or suitable combinations of the results yield oscillation criteria for this equation. The novelty of the presented results is in the fact that we used parametrized versions of inequalities used typically in comparison theory of neutral differential equations. Despite the fact that we introduced three parameters $(l, \varphi$, and $\eta)$, the results remain simple and effective. We have shown on several examples that effective oscillation criteria can be formulated for particular equations by establishing the optimal values for these parameters.

\section{Conflict of Interests}

The authors declare that there is no conflict of interests regarding the publication of this paper.

\section{Acknowledgments}

The authors would like to thank the referee for valuable comments to the paper. Research supported by the Grant P201/10/1032 of the Czech Science Foundation.

\section{References}

[1] B. Baculíková and J. Džurina, "Oscillation theorems for second order neutral differential equations," Computers \& Mathematics with Applications, vol. 61, no. 1, pp. 94-99, 2011.

[2] B. Baculíková and J. Džurina, "Oscillation theorems for secondorder nonlinear neutral differential equations," Computers \& Mathematics with Applications, vol. 62, no. 12, pp. 4472-4478, 2011.

[3] B. Baculíková, T. Li, and J. Džurina, "Oscillation theorems for second order neutral differential equations," Electronic Journal 
of Qualitative Theory of Differential Equations, no. 74, pp. 1-13, 2011.

[4] B. Baculíková, T. Li, and J. Džurina, "Oscillation theorems for second-order superlinear neutral differential equations," Mathematica Slovaca, vol. 63, no. 1, pp. 123-134, 2013.

[5] T. Li, "Comparison theorems for second-order neutral differential equations of mixed type," Electronic Journal of Differential Equations, vol. 2010, no. 167, pp. 1-7, 2010.

[6] J.-G. Dong, "Oscillation behavior of second order nonlinear neutral differential equations with deviating arguments," Computers \& Mathematics with Applications, vol. 59, no. 12, pp. 37103717, 2010.

[7] S. Fišnarová and R. Mařík, "Oscillation of half-linear differential equations with delay," Abstract and Applied Analysis, vol. 2013, Article ID 583147, 6 pages, 2013.

[8] M. Hasanbulli and Y. V. Rogovchenko, "Oscillation criteria for second order nonlinear neutral differential equations," Applied Mathematics and Computation, vol. 215, no. 12, pp. 4392-4399, 2010.

[9] R. P. Agarwall, M. Bohner, T. Li, and Ch. Zhuang, "Oscillation of second order Emden-Fowler neutral delay differential equations," in Annali di Matematica Pura ed Applicata, pp. 1-15, 2013.

[10] Z. Han, T. Li, S. Sun, and Y. Sun, "Remarks on the paper," Applied Mathematics and Computation, vol. 207, pp. 388-396, 2009, Applied Mathematics and Computation, vol. 215, pp. 39984007, 2010.

[11] T. Li, Z. Han, Ch. Zhang, and H. Li, "Oscillation criteria for second-order superlinear neutral differential equations," Abstract and Applied Analysis, vol. 2011, Article ID 367541, 17 pages, 2011.

[12] T. Li, Y. V. Rogovchenko, and Ch. Zhang, "Oscillation of secondorder neutral differential equations," Funkcialaj Ekvacioj, vol. 56, no. 1, pp. 111-120, 2013.

[13] Y. G. Sun and F. W. Meng, "Note on the paper of Džurina and Stavroulakis," Applied Mathematics and Computation, vol. 174, no. 2, pp. 1634-1641, 2006.

[14] S. Sun, T. Li, Z. Han, and Y. Sun, "Oscillation of secondorder neutral functional differential equations with mixed nonlinearities," Abstract and Applied Analysis, vol. 2011, Article ID 927690, 15 pages, 2011.

[15] S. Sun, T. Li, Z. Han, and H. Li, "Oscillation theorems for second-order quasilinear neutral functional differential equations," Abstract and Applied Analysis, vol. 2012, Article ID 819342, 17 pages, 2012.

[16] L. Ye and Z. Xu, "Oscillation criteria for second order quasilinear neutral delay differential equations," Applied Mathematics and Computation, vol. 207, no. 2, pp. 388-396, 2009.

[17] J. R. Graef and S. H. Saker, "New oscillation criteria for generalized second-order nonlinear neutral functional differential equations," Dynamic Systems and Applications, vol. 19, no. 3-4, pp. 455-472, 2010.

[18] S. Fišnarová and R. Mařík, "Oscillation criteria for neutral second-order half-linear differential equations with applications to Euler type equations," Boundary Value Problems, vol. 2014, article 83, 2014.

[19] R. P. Agarwall, S. R. Grace, and D. O'Regan, Oscillation Theory for Difference and Functional Differential Equations, Kluwer Academic Publishers, Dordrecht, The Netherlands, 2000.

[20] R. G. Koplatadze and T. A. Chanturiya, "Oscillating and monotone solutions of first-order differential equations with deviating argument," Differentsial'nye Uravneniya, vol. 18, no. 8, pp. 1463-1472, 1982 (Russian).
[21] Y. Kitamura and T. Kusano, "Oscillation of first-order nonlinear differential equations with deviating arguments," Proceedings of the American Mathematical Society, vol. 78, no. 1, pp. 64-68, 1980. 


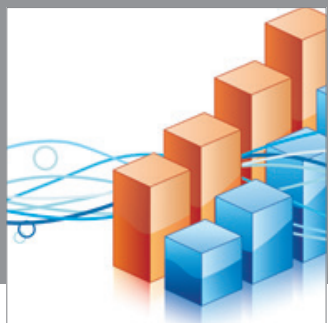

Advances in

Operations Research

mansans

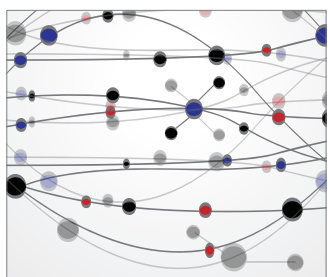

The Scientific World Journal
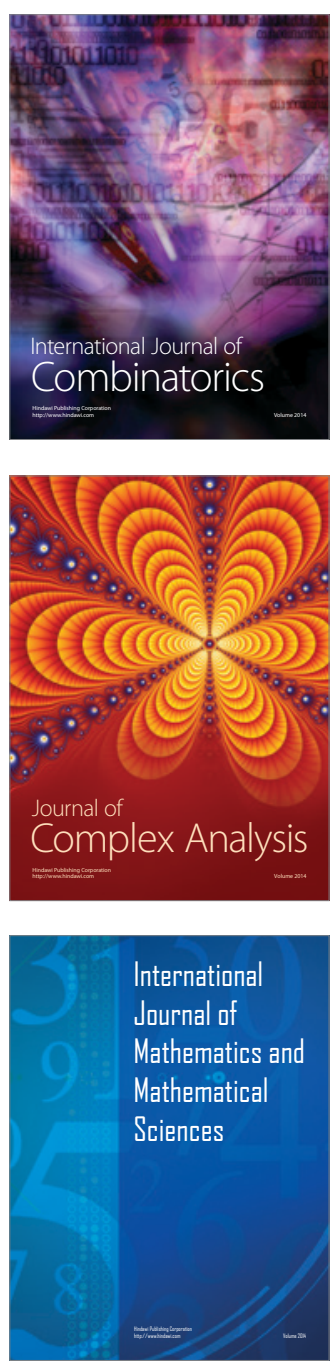
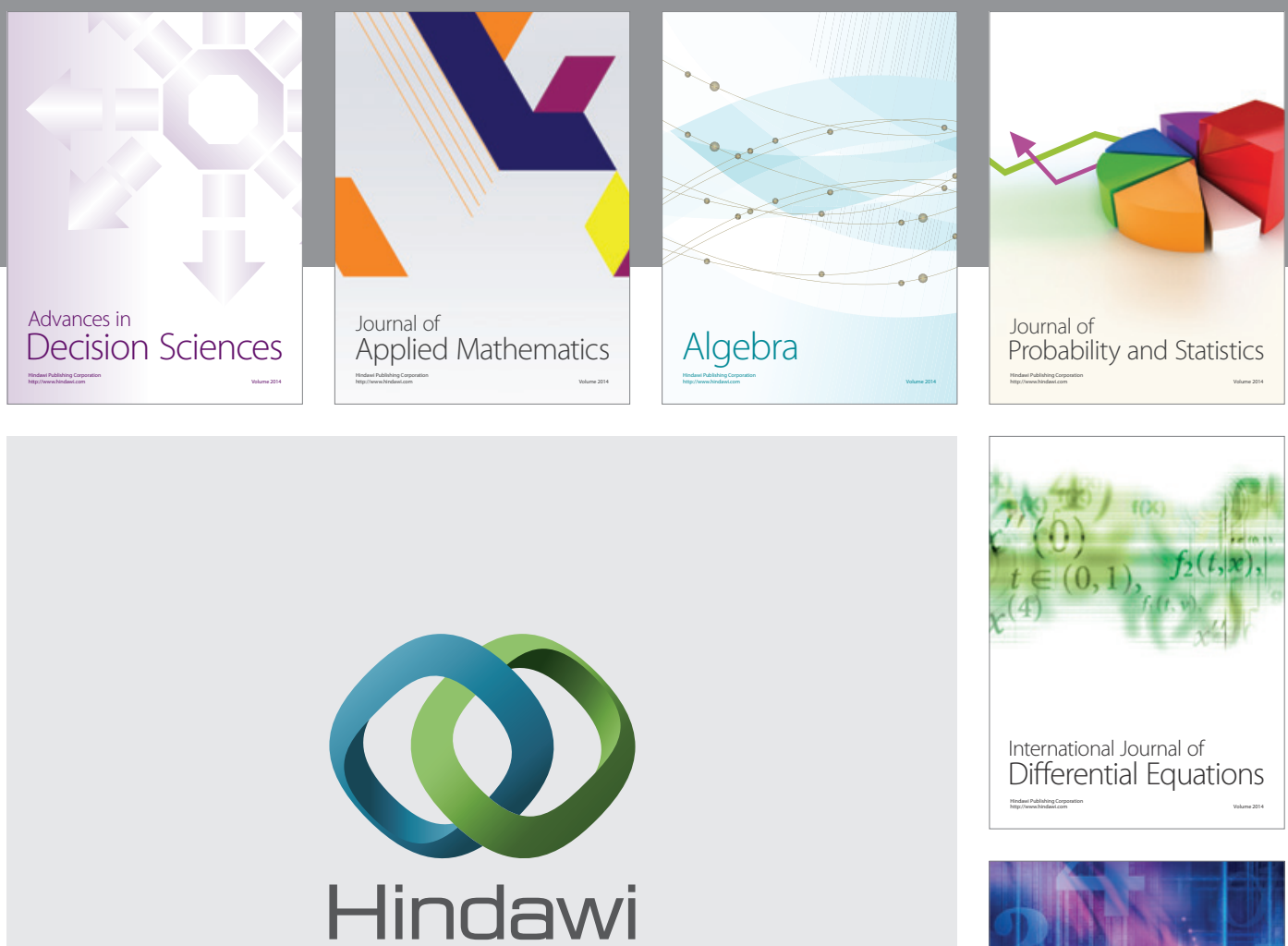

Submit your manuscripts at http://www.hindawi.com
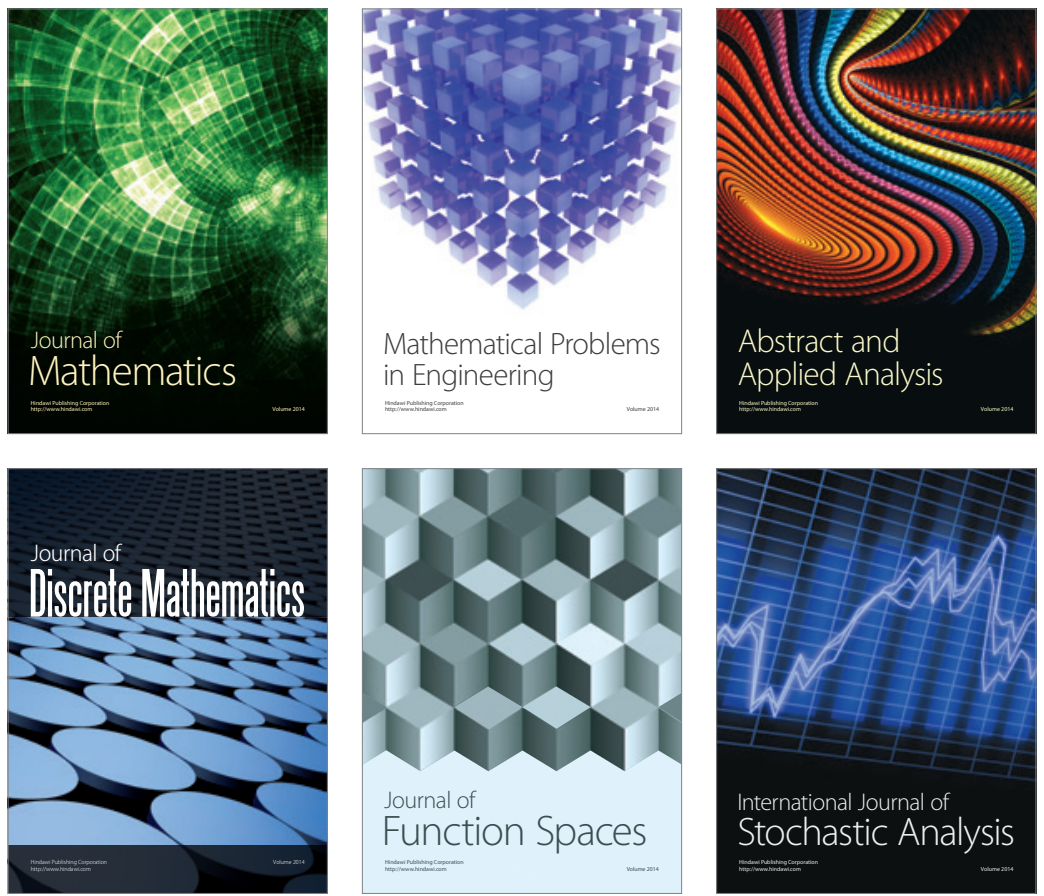

Journal of

Function Spaces

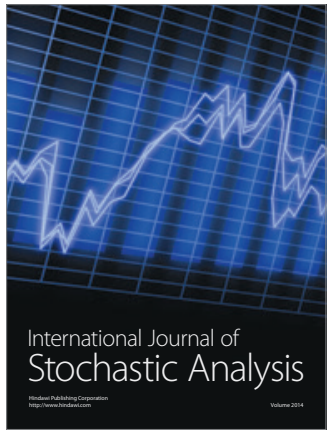

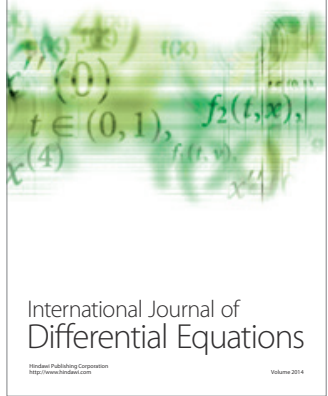
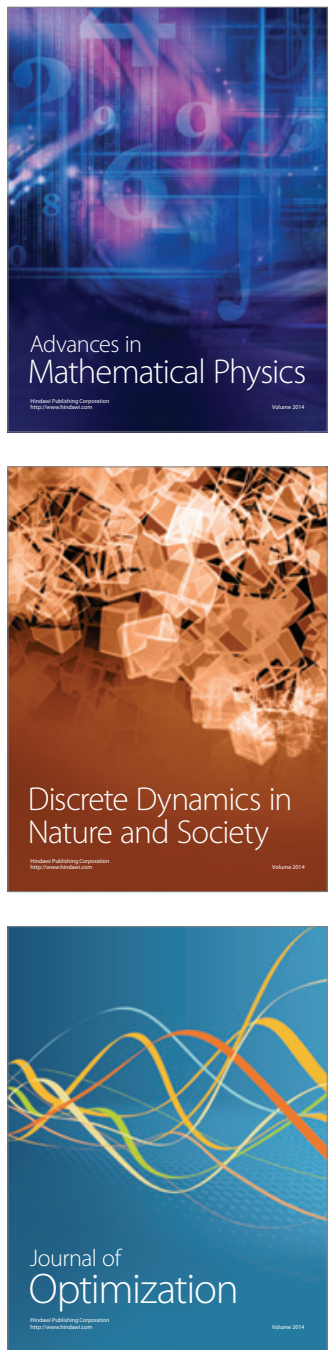